\title{
AVALIAÇÃO DE SISTEMAS DE INFORMAÇÃO: REVISÃO DA LITERATURA'
}

\section{EVALUATION OF INFORMATION SYSTEMS: LITERATURE REVIEW}

\author{
Osmar Arouck ${ }^{2}$
}

\begin{abstract}
RESUMO
Revisão da literatura sobre avaliação de sistemas de informação. Analisa 104 artigos publicados em 14 diferentes periódicos entre 1974 a 1997. Identifica, analisa e sistematiza as variáveis dependentes adotadas para avaliação de sistemas de informação ao longo deste periodo. Enfoca especificamente os atributos referentes à qualidade da informação e qualidade de serviço em sistemas de informação. Apresenta a literatura referente à utilização do instrumento da área de marketing de serviço chamado SERVQUAL, bem como sua aplicação na área de sistemas de informação.
\end{abstract}

Palavras-chave: satisfação do usuário da informação; mensuração da satisfação do usuário; sucesso de sistemas de informação; avaliação de sistemas de informação; qualidade da informação; gestão de sistemas de informação.

\section{ABSTRACT}

Literature review on information systems assessment. It analyses 104 published articles in 14 different periodicals between 1974 and 1997. It identifies, analyses, and systematizes dependent variables adopted for evaluating information systems through that period. It focuses specifically the attributes related to the information quality and service quality of information systems. It also presents the literature referring to the using of the service marketing area instrument SERVQUAL, as well as its applicability in the information systems field.

Key words: user information satisfaction; measures of user satisfaction; information systems success; information systems assessment; information quality; management information systems.

\section{INTRODUÇÃO}

Peter Keen (1980) identifcou cinco pontos para estabelecer a área de gestão de sistemas de informação como um campo de pesquisa coerente e maduro:

- definir as disciplinas de referência;

- defnir as variáveis dependentes;

\footnotetext{
(1) Pesquisa realizada para redação de Disscrtaçào de Mestrado cm Informática na Université du Québec à Montréal (UQAM), Canadá, patrocinada pela Agence Canadienne de Dévelopement Intenational (ACDI). O autor agradece a orientação do Dr. Marc Bouisset da UQAM.

(2) Professor do Departamento de Biblioteconomia da Universidade Federal do Pará. E-mail: arouck@ufpa.br
} 
- estabelecer uma tradição cumulativa;

- identifcar a natureza do relacionamento entre a pesquisa e a prática em gestão de sistemas de informação e a tecnologia de informática;

- identificar os meios de difusão do conhecimento para publicar resultados de pesquisa na área (Keen,1980).

Este trabalho busca contribuir na identificação e sistematização de variáveis dependentes relacionadas à avaliação de sistemas de informação, no aspecto específico de atributos de qualidade da informação, bem como identifcar métodos de avaliação destes atributos.

Um dos objetivos principais da gestão de sistemas de inforrnação é desenvolver, operar e manter sistemas de informação que contribuam para que a organização a que estão relacionados cumpra seus objetivos (Hamilton e Chervany, 1981). O cumprimento destes objetivos pode ser avaliado a partir de duas perspectivas:

a) a eficiência com que o sistema utiliza os recursos disponiveis (pessoal, equipamentos, orçamento) para prover às necessidades dos usuários;

b) a eficácia no uso do sistema de informação na ação dos usuários individuais ou da organização, para que estes realizem sua tarefa dentro da missão da organização (Hamilton e Chervany, 1981).

A avaliação de sistemas de informação é uma necessidade para o gestor, tanto para a melhoria destes sistemas, como para justificar os altos investimentos realizados neste setor (Myers, Kappelman e Prybutok, 1997; Brancheau, Janz e Wetherbe, 1996; Galletta e
Lederer,1989; Dickson e Wetherbe,1983; Hamilton e Chervany, 1981).

Apesar da sentida necessidade de avaliação de sistemas de informação, não há na literatura uma definição clara e objetiva dos métodos a serem empregados. A falta de padronização dos métodos não permite a consolidação de dados para estudos comparativos (Myers, Kappelman e Prybutok,1997; DeLone e McLean, 1992; Saunders e Jones, 1992; Jarvenpaa, Dickson e DeSanctis, 1985; Dickson et al.,1984).

\section{Interesse da pesquisa em avaliação de sistemas de informação}

Desde 1980, a "Society for Information Management”(SIM) e o "MIS Research Center"(MISRC) realizam periodicamente pesquisas para determinar as questões mais críticas (enjeux plus critiques) na área de gestão de sistemas de informação. Os resultados destas pesquisas foram publicados no periódico "MIS Quarterly". Dentre os problemas identificados nestas pesquisas, a avaliação da eficácia de sistemas de informação esteve sempre presente. Nos anos 80 esta questão manteve-se entre as dez mais importantes, caindo para $16^{\circ}$ lugar em 1990 ; tendo subido para o $11^{\circ}$ lugar na última pesquisa, conforme pode ser visto na Tabela 1, abaixo.

A queda para $16^{\circ}$ lugar do item avaliação de eficácia de sistemas de informação, em 1990, surpreendeu os autores daquela pesquisa (Niederman, Brancheau e Wetherbe, 1991). Eles argumentam que esta queda se deve ao fato de que o papel de tecnologia de informação

Tabela 1. Eficácia de sistemas de informação nas pesquisas SIM/MIRSC.

\begin{tabular}{|c|c|c|c|}
\hline Ano & Classificação & Autores & Expressão adotada \\
\hline 1980 & $2^{\circ}$ & Ball e Harris, 1982 & gauging MIS effectiveness \\
\hline 1983 & $5^{\circ}$ & Dickson et al., 1984 & $\begin{array}{l}\text { measuring and improving IS } \\
\text { effectiveness/productivity }\end{array}$ \\
\hline 1986 & $9^{\circ}$ & Brancheau e Wetherbe, 1987 & measuring effectiveness \\
\hline 1990 & $16^{\circ}$ & $\begin{array}{l}\text { Niederman, Brancheau e Wetherbe, } \\
\text { Brancheau e Wetherbe, } 1991\end{array}$ & IS effectiveness measurement \\
\hline $1994-95$ & $11^{\circ}$ & Brancheau, Janz e Wetherbe, 1996 & $\begin{array}{l}\text { measuring IS effectiveness and } \\
\text { productivity }\end{array}$ \\
\hline
\end{tabular}

Fonte: Arouck, 1999 
no desempenho empresarial é sutil e difícil de se desenredar de outros fatores.

Algumas tentativas de se estabelecer medidas de avaliação mostraram-se ineficientes e desencorajaram algumas pesquisas. As medidas de eficácia de sistemas de informação precisam ser vinculadas ao desempenho organizacional, e estas medidas devem ser fidedignas e de veriftcação continuada. Uma questão chave no futuro, segundo os autores, será determinar como medir os impactos de investimento de tecnologia de informação na organização (Niederman, Brancheau e Wetherbe, 1991).

Na última avaliação, o item referente a aferição da eflcácia de sistemas de informação subiu para $11^{\circ}$ lugar. $\mathrm{O}$ fato é analisado como conseqüência da necessidade de se justifcar os novos investimentos realizados nos sistemas de informação (Brancheau, Janz e Wetherbe, 1996; cf. Dickson e Wetherbe, 1983).

Os autores constatam que avaliação de sistemas de informação é um problema que vem se mantendo sem resolução eficiente. É uma questão antiga e que apresenta muitas dificuldades para se estabelecer métodos fidedignos e objetivos. Enquanto o valor dos custos para implantação de sistemas de informação é de fácil estabelecimento, os benefícios oriundos destes são difíceis de avaliar e medir. Historicamente, não se tem tido muito sucesso ao se tentar medir o impacto de sistemas de informação no lucro ou desempenho das organizações. Avaliar sistemas de informação é difícil, mas necessário (cf. Brancheau, Janz e Wetherbe, 1996; Niederman, Brancheau e Wetherbe, 1991; Brancheau e Wetherbe,1987; Dickson et al.,1984; Ball e Harris, 1982).

\section{FONTES CONSULTADAS}

\section{Autores principais}

Diversos modelos teóricos para avaliação de sistemas de informação foram propostos na literatura. Entretanto a maioria dos trabalhos indica os atributos a avaliar, sem definir claramente o modo que estas características devem ser avaliadas (Myers, Kappelman e Prybutok, 1997). Os trabalhos de Myers, Kappelman e Prybutok (1997), Grover, Jeong e Segars (1996), DeLone e McLean (1992) e de Saunders et Jones (1992) são os mais recentes e também os mais exaustivos.

Esta revisão partiu de algumas outras revisões dos últimos dez anos: Galletta e Lederer (1989), Conrath e Mignen (1990), DeLone e McLean (1992), Myers, Kappelman e Prybutok (1997), Grover, Jeong e Segars (1996), bem como a revisão de Asubonteng, McCleary e Swan (1996) sobre qualidade de serviço a partir do trabalho de Parasuraman, Zeithaml e Berry (1985, 1988).

Galletta e Lederer (1989) verificaram a fidedignidade dos resultados obtidos na avaliação da satisfação dos usuários de sistemas de informação, utilizando o instrumento de 13 itens proposto por Ives, Olson e Baroudi (1983), que se baseia naquele de 39 itens proposto por Bailey e Pearson (1983). Neste trabalho os autores concluem que o instrumento proposto não possui fidedignidade suficiente, e que se deve ter cuidado na interpretação dos dados obtidos através desta metodologia. Os autores afirmam também a necessidade de se ter um instrumento menos heterogêneo e com maior fidedignidade para avaliação de sistemas de informação.

Conrath e Mignen (1990) constataram que, apesar de haver diferentes metodologias de avaliação da satisfação de usuários de sistemas de informação, poucas organizações avaliavam sistematicamente a satisfação de seus usuários. Este trabalho levantou dados sobre métodos adotados, periodicidade e motivação das avaliações.

DeLone e McLean (1992) sistematizaram os trabalhos na áreas de avaliação de sistemas de informação, propondo seis dimensões para agrupar os diferentes fatores analisados na literatura. Este modelo e o trabalho de Saunders et Jones (1992) serviram de base para o trabalho de Myers, Kappelman et Prybutok (1997).

Grover, Jeong e Segars (1996) realizaram sua revisão de literatura enfocando medidas de eficácia de sistemas de informação, organizando-a em quatro perspectivas: bases teóricas para a definição de critérios de eficácia estatística e avaliação de medidas para determinação de eficácia, relacionamentos entre os critérios de eficácia e outras variáveis, e elementos que influenciam a eficácia de sistemas de informação.

Considerando-se que a dimensão qualidade de serviço é adicionada ao modelo de DeLone e McLean (1992) a partir do trabalho de Myers, Kappelman et Prybutok (1997), acrescentou-se a revisão de literatura sobre esta dimensão, a partir de Asubonteng, McCleary e Swan (1996). Trata-se de uma revisão crítica do modelo proposto por Parasuraman, Zeithaml e Berry $(1985,1988)$ que é um instrumento bastante utilizado na área de marketing, chamado SERVQUAL. 


\section{PERIÓDICOS CONSULTADOS}

Utilizaram-se os periódicos identificados por Hamilton e Ives (1983), por DeLone e McLean (1992) e Grover, Jeong e Segars (1996), excluindo-se apenas Transactions on Database Systems, acrescidos de outros que foram identificados na pesquisa realizada em bases bibliográficas especializadas e nas bibliotecas. Este núcleo de periódicos é identificado para estudos analíticos similares e é considerado como referência para a publicação de resultados de pesquisas semelhantes ao presente trabalho. Os periódicos utilizados nesta revisão figuram na Tabela 2 , abaixo, na qual se indica o número total de artigos consultados, agrupados por períodos.

A pesquisa original deste trabalho não trabalhou com periódicos de língua portuguesa. Para a edição deste artigo, a título de indicação de leitura, acrescentamos duas referências em língua portuguesa relacionados a este tema (Morgado e Watson, 1998; Freitas et al, 1994).

Tabela 2. Periódicos consultados na revisão da literatura.

\begin{tabular}{|c|c|c|c|c|}
\hline \multirow{2}{*}{ Periódicos } & \multicolumn{4}{|c|}{ Número de artigos consultados } \\
\hline & 1974-1979 & 1980-1989 & 1990-1997 & Total \\
\hline MIS Quarterly. & & 13 & 11 & 24 \\
\hline Journal of Marketing & & 3 & 10 & 13 \\
\hline Decision Sciences & 1 & 6 & 4 & 11 \\
\hline Journal of Retailing & & 2 & 7 & 9 \\
\hline Information \& Management & 1 & 2 & 6 & 9 \\
\hline Journal of MIS & & 1 & 7 & 8 \\
\hline Management Science & 2 & 4 & 2 & 8 \\
\hline The Journal of Service Marketing & & 1 & 5 & 6 \\
\hline Information Resources Management Journal & & & 4 & 4 \\
\hline Sloan Management Review & & & 4 & 4 \\
\hline Comunication of the ACM & & 3 & & 3 \\
\hline ICIS Proceedings & & 1 & 2 & 3 \\
\hline Information System Research & & & 1 & 1 \\
\hline Academy of Management Journal & 1 & & & 1 \\
\hline Total & 5 & 36 & 63 & 104 \\
\hline
\end{tabular}

Fontc: Arouck, 1999

\section{AVALIAÇÃO DE SISTEMAS DE INFORMAÇÃO}

Os estudos na área de avaliação de sistemas de informação têm seu marco referencial em alguns trabalhos que são citados regularmente pelos pesquisadores da área: Bailey e Pearson (1983); Ives, Olson e Baroudi (1983); Baroudi e Orlikowski (1988); DeLone e McLean (1992). Os três primeiros trabalham com a satisfação do usuário, propondo instrumento de verificação da mesma. DeLone e McLean (1992) identificaram na literatura as diferentes variáveis dependentes que são utilizadas na avaliação de sistemas de informação, esta revisão compreende o período de 1981 a 1987.
Dois artigos anteriores ao trabalho de DeLone e McLean (1992) realizaram extensa revisão de literatura no tema: Zmud (1979); Ives e Olson (1984). Zmud (1979, 1978) considerou três categorias para o sucesso de um sistema de informação: desempenho do usuário, uso do sistema, satisfação do usuário ${ }^{3}$. Ives e Olson (1984) adotaram duas classes de variáveis: qualidade do sistema e aceitação do sistema; sendo que em aceitação do sistema incluiu-se o uso do sistema, impacto do sistema no comportamento do usuário e satisfação com a informação $0^{4}$. Ambos os trabalhos, no entanto, preocuparam-se com a análise de variáveis independentes, isto é, envolvimento do usuário, no

\footnotetext{
(3) user performance, MIS usage, user satisfaction.

(4) system usage, system impact on user behavior, and information satisfaction
} 
caso de Ives e Olson, e diferenças individuais no estudo de Zmud; enquanto que no trabalho de DeLone e McLean o enfoque recai sobre as variáveis dependentes (DeLone e McLean, 1992).

O modelo apresentado para identificar as dimensões e fatores para avaliação de sistemas de informação de DeLone e McLean (1992) é baseado no trabalho de Richard Mason (1978) que, por sua vez, é estruturado sobre a teoria matemática da comunicação de Shannon e Weaver (1949).

Warren Weaver, a partir da teoria matemática da comunicação de Claude Shannon (1949) classificou os problemas da comunicação em três níveis hierárquicos: nível técnico, nível semântico e nível de efcácia ou influência. O problema técnico diz respeito à acuidade na transmissão dos símbolos de comunicação. $\mathrm{Na}$ dimensão semântica, atenta-se à precisão da transmissão destes símbolos em relação ao sentido desejado. E, finalmente, temos o nível de eficácia que diz respeito ao impacto desta comunicação no comportamento dos indivíduos envolvidos no processo.
Baseados nos trabalhos de Shannon e Weaver (1949) e de Mason (1978), DeLone e McLean (1992) agruparam as variáveis independentes estudadas nos diferentes trabalhos realizados na área de qualidade de sistemas de informação. Leyland Pitt, Richard Watson e C. Kavan (1995; cf. Pitt e Watson, 1994) acrescentam a categoria qualidade de serviço ao modelo proposto por DeLone e McLean (1992); inclui-se ainda a dimensão impacto no grupo de trabalho, proposta por Myers, Kappelman e Prybutok (1997), conforme se pode ver na Tabela 3, abaixo.

A interdependência das dimensões - demonstrada na figura 1 - foi proposta inicialmente por DeLone e McLean (1992), ampliada por Myers, Kappelman e Prybutok (1997): a qualidade do sistema, a qualidade da informação e a qualidade do serviço, individualmente e conjuntamente, influenciam a utilização do sistema e a satisfação do usuário. A freqüência do uso pode afetar, positiva ou negativamente, o grau de satisfação do usuário, e vice-versa. A utilização do

Tabela 3. Niveis e dimensões para avaliação de sistemas de informação.

\begin{tabular}{|c|c|c|c|c|}
\hline Autor & Ano & \multicolumn{3}{|c|}{ Níveis de dimensões } \\
\hline Shannon e Weaver & 1949 & Nível técnico & Nível semântico & Nível de eficácia, ou impacto \\
\hline Mason & 1978 & - produção & - produção & $\begin{array}{l}\text { - recepção } \\
\text { - influência no receptor } \\
\text { - influência no sistema }\end{array}$ \\
\hline DeLone e McLean & 1992 & - qualidade do sistema & - qualidade do sistema & $\begin{array}{l}\text { - uso } \\
\text { - satisfação do usuário } \\
\text { - impacto individual } \\
\text { - impacto organizacional }\end{array}$ \\
\hline Pitt, Watson e Kavan & 1995 & - qualidade do sistema & - qualidade do sistema & $\begin{array}{l}\text { - uso } \\
\text { - satisfação do usuário } \\
\text { - impacto individual } \\
\text { - impacto organizacional }\end{array}$ \\
\hline Myers, Kappelman e Prybutok & 1997 & - qualidade de sistema & - qualidade de sistema & $\begin{array}{l}\text { - uso } \\
\text { - satisfação do usuário } \\
\text { - impacto individual } \\
\text { - impacto no grupo de trabalho } \\
\text { - impacto organizacional }\end{array}$ \\
\hline
\end{tabular}

Fontc: Arouck, 1999 
sistema e a satisfação do usuário são antecedentes diretos do impacto individual; este impacto no desempenho individual pode influenciar no desempenho do grupo de trabalho e no ambiente organizacional. O impacto no grupo de trabalho pode também causar modificações no ambiente organizacional. (Myers,
Kappelman e Prybutok,1997; DeLone e McLean, 1992). Seddon e Kiew (1994) examinaram empiricamente este inter-relacionamento nas dimensões qualidade do sistema, qualidade da informação, uso e satisfação do usuário, confirmando parcialmente a proposta de DeLone e McLean (1992).

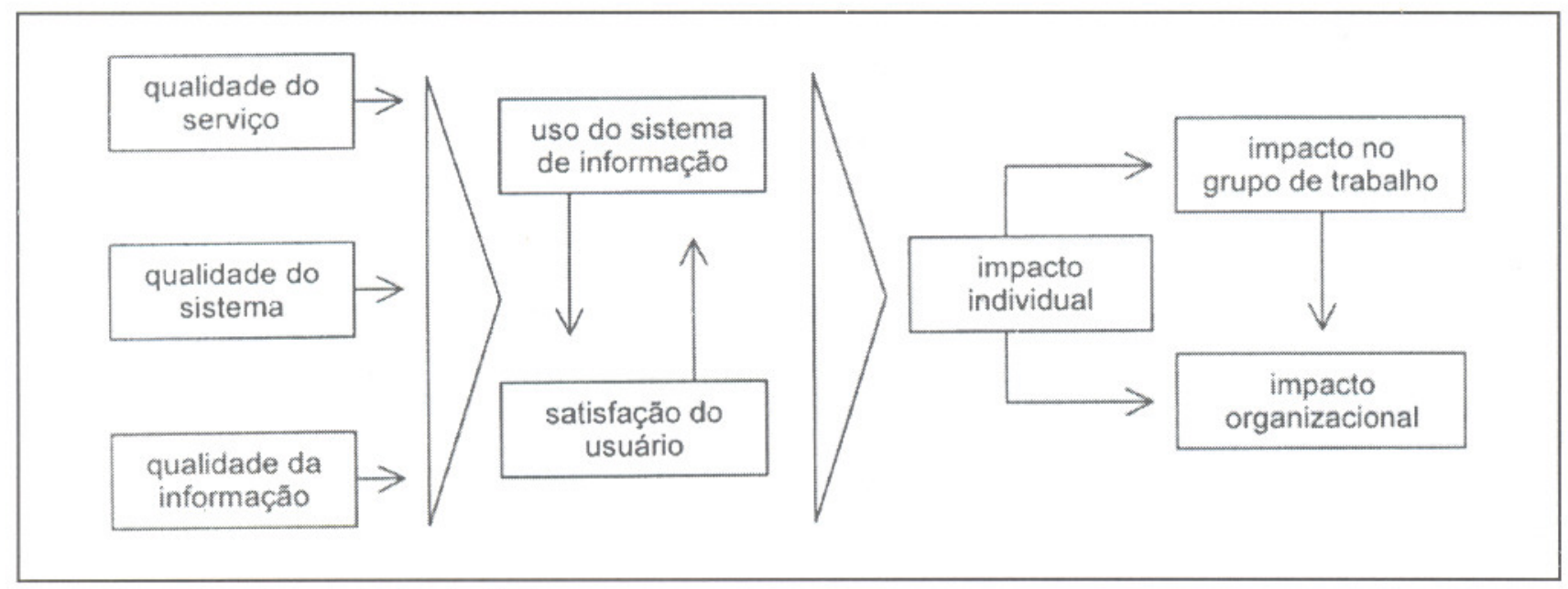

Fonte: Myres, Kappelman c Prybutok, 1997

Figura 1. Dimensões de sistema de informação e seus relacionamentos.

\section{NÍVEIS, DIMENSÕES E CARACTERÍSTICAS PARA AVALIAÇÃO DE SISTEMAS DE INFORMAÇÃO}

Consideramos para este trabalho como níveis aqueles propostos por Shannon e Weaver (1949) compreendendo sua complementariedade e mútua influência. Como dimensão identificamos as seis categorias identificadas por DeLone e McLean (1992), acrescidas daquelas introduzidas por Myers, Kappelman e Prybutok (1997) e Pitt, Watson e Kavan (1995). Dentro de cada dimensão há diferentes características que são propriedades ou atributos que podem ser identificados como variáveis dependentes na avaliação de sistemas de informação, e que por sua vez podem ser organizadas em subdimensões.

Deste modo, temos no nível técnico a qualidade do sistema; no nível semântico

temos qualidade da informação e qualidade de serviço; e no nível de efcácia temos uso do sistema, satisfação do usuário, impacto individual, impacto no grupo de trabalho e impacto organizacional (ver Tabela 3 , acima).

$\mathrm{Na}$ dimensão de qualidade do sistema, Mason (1978) identifica o sistema de produção de informação como uma conjunto de operações que recebe e processa dados a partir de regras e procedimentos precisos e sistemáticos. DeLone e McLean (1992) destacaram as seguintes características nesta dimensão: acessibilidade, flexibilidade, integração, tempo de resposta, confabilidade, facilidade de uso, utilidade.

Em um sistema de informação, o nível de impacto é o mais difícil de ser definido e avaliado de forma objetiva e não ambígua (DeLone e McLean, 1992). Uma outra dificuldade apontada é o fato de se estabelecer quais os efeitos causados num receptor oriundos do sistema de informação e quais os provenientes de outra fonte de influência (Mason,1978).

No uso do sistema são identiflcadas características como tempo de quantidade de acessos, forma de utilização, usuários, e nível de utilização (DeLone e McLean,1992).

A dimensão satisfação do usuário é a medida mais usada em avaliação de sistemas de informação. O instrumento proposto por Bailey e Pearson (1983) contribui para o uso regular desta dimensão, dada a sua facilidade de aplicação, segundo DeLone e McLean (1992). Nesta 
dimensão, identificam-se, dentre outros, os seguintes fatores: satisfação com características específicas, satisfação total, satisfação com a informação, satisfação com software, satisfação em relação à tomada de decisão (DeLone McLean, 1992).

$\mathrm{Na}$ dimensão impacto individual, DeLone e McLean (1992) agruparam compreensão da informação, aprendizagem, eficácia da decisão, melhora da produtividade individual, modificação da decisão, e identificação e solução de problemas.

$\mathrm{Na}$ dimensão impacto organizacional DeLone e McLean (1992) reuniram a valorização patrimonial, aumento dos lucros, redução de custos de funcionamento, redução de pessoal, ganhos de produtividade, aumento das vendas, crescimento do mercado, melhora da relação entre custo e benefício (DeLone e McLean, 1992).

A influência do sistema de informação no grupo de trabalho, como elemento intermediário entre o indivíduo e a organização é proposto por Myers, Kappelman e Prybutok (1997) e Ishman (1996), considerando a importância que as equipes e grupos de trabalhos têm tido no ambiente organizacional.

Neste trabalho enfoca-se a dimensão qualidade da informação, adotando-se uma metodologia de avaliação oriunda da área de qualidade de serviço. Deste modo, a revisão de literatura enfocou estas duas dimensões, conforme é apresentado a seguir.

\section{QUALIDADE DA INFORMAÇĀO}

Compreende-se nesta dimensão o produto, a informação fornecida por um sistema de informação. $\mathrm{Na}$ pesquisa de DeLone e McLean (1992) são analisados nove artigos da década de oitenta (1983-1987) referentes a esta dimensão. Neste trabalho foram analisados os nove da revisão de DeLone e McLean (1992) e mais outros dez, perfazendo dezenove artigos do período de 1974 a 1997. Os autores, ano de publicação e as características analisadas nos artigos encontram-se na Tabela 4, abaixo.

Tabela 4. Artigos referentes à dimensão qualidade de informação.

\begin{tabular}{|c|c|}
\hline Autores e ano & Caracteristicas \\
\hline Gallagher, 1974 & $\begin{array}{l}\text { disposição, arranjo, legibilidade, clareza, exatidão, confiabilidade, veracidade, } \\
\text { validade, oportunidade, atualização, completude, suficiência }\end{array}$ \\
\hline Swanson, 1974 & $\begin{array}{l}\text { legibilidade, clareza, exatidão, oportunidade, concisão, singularidade, } \\
\text { compreensibilidade, relevância }\end{array}$ \\
\hline Zmud, 1978 & $\begin{array}{l}\text { disposição, arranjo, legibilidade, clareza, exatidão, confiabilidade, veracidade, } \\
\text { validade, oportunidade, atualização, qualidade, completude, suficiência, eficácia, } \\
\text { compreensibilidade, relevância, importância, utilidade, aplicabilidade }\end{array}$ \\
\hline Ahituv, 1980 & $\begin{array}{l}\text { relevância } \\
\text { oportunidade, tempode resposta, exatidão } \\
\text { concepção gráfica, meio, disposição, arranjo }\end{array}$ \\
\hline Larcker e Lessig, 1980 & $\begin{array}{l}\text { importância, relevância valor informativo, valor instrutivo, utilidade } \\
\text { oportunidade, exatidão } \\
\text { clareza, legibilidade }\end{array}$ \\
\hline Olson e Lucas, 1982 & $\begin{array}{l}\text { exatidão } \\
\text { aparência }\end{array}$ \\
\hline
\end{tabular}


cont.

\begin{tabular}{|c|c|}
\hline Autores e ano & Características \\
\hline Bailey e Pearson, 1983 & $\begin{array}{l}\text { relevância } \\
\text { exatidão, completude, atualização, exatidão, confiabilidade, oportunidade, volume } \\
\text { representação }\end{array}$ \\
\hline King e Epstein, 1983 & $\begin{array}{l}\text { relevância, valor instrutivo } \\
\text { confiabilidade, quantidade, oportunidade, suficiência }\end{array}$ \\
\hline Blaylock e Rees, 1984 & importância, utilidade \\
\hline Mahmood e Medewitz, 1985 & $\begin{array}{l}\text { utilidade } \\
\text { exatidão }\end{array}$ \\
\hline Rivard e Huff, 1985 & utilidade \\
\hline Srinivasan, 1985 & $\begin{array}{l}\text { utilidade, relevância, valor instrutivo } \\
\text { exatidão, adequação, oportunidade } \\
\text { aparência, representação, disposição, arranjo }\end{array}$ \\
\hline Jones e McLeod, 1986 & importância \\
\hline Iivari e Koskela, 1987 & $\begin{array}{l}\text { valor informativo, inteligibilidade } \\
\text { completude, atualização, confiabilidade, validade, tempo de resposta, } \\
\text { oportunidade legibilidade, representação }\end{array}$ \\
\hline Miller e Doyle, 1987 & $\begin{array}{l}\text { relevância } \\
\text { exatidão, completude, oportunidade, volume }\end{array}$ \\
\hline Rainer e Watson, 1995 & $\begin{array}{l}\text { relevância, adequação } \\
\text { exatidão, concisão, oportunidade }\end{array}$ \\
\hline Palvia, 1996 & $\begin{array}{l}\text { relevância, adequação, utilidade } \\
\text { exatidão, tempo de resposta, atualização clareza }\end{array}$ \\
\hline Saarinen, 1996 & $\begin{array}{l}\text { relevância } \\
\text { exatidão, completude, confiabilidade, oportunidade, atualização } \\
\text { clareza, representação }\end{array}$ \\
\hline Klein, Goodhue e Davis, 1997 & exatidão, completude, atualização, consistência \\
\hline
\end{tabular}

Fontc: Arouck, 1999

\section{QUALIDADE DO SERVIÇO}

A literatura da área de marketing de serviço é uma fonte confiável para empréstimos metodológicos de procedimentos de avaliação de desempenho de sistemas de informação (Kettinger e Lee, 1994).

$\mathrm{Na}$ área de marketing de serviço, Parasuraman, Zeithaml e Berry desenvolveram um modelo conceptual (Gap model) bem aceito entre os pesquisadores e gestores; identificaram as variáveis determinantes de qualidade de serviço; bem como apresentaram um instrumento para medir a qualidade de serviço (SERVQUAL) consolidando o marketing de serviço como um campo de estudos e pesquisa sólido (Asubonteng, McCleary e Swan, 1996; Fisk, Brown e Bitner, 1993; cf. Berry e Parasuraman,1991,1992,1993; Berry, Parasuraman e Zeithaml, 1984, 1988, 1993, 1994; Berry, Zeithaml e Parasuraman,1990; Parasuraman e Berry,1991; Parasuraman, Berry eZeithaml, 1990, 1991a, 1991b, 1991c, 1993; Parasuraman, Zeithaml e Berry, 1985, 1988, 1994a, 
1994b, 1994c; Zeithaml, Berry e Parasuraman, 1988, 1991, 1993, 1996; Zeithaml, Parasuraman e Berry, 1985, 1990).

\section{O MODELO DE PARASURAMAN, ZEITHAML E BERRY (GAP MODEL)}

Parasuraman, Zeithaml e Berry (1985), baseando-se em uma pesquisa exploratória, iniciaram uma pesquisa sobre qualidade de serviço e seus determinantes. Este grupo de pesquisa define qualidade de serviço como o grau de discrepância entre as expectativas de serviço e a percepção do serviço prestado. Os autores identificaram um conjunto de atributos que os usuários adotam como critério para avaliar o desempenho do serviço.

$\mathrm{Na}$ Figura 2, abaixo, tem-se o gráfico representativo do modelo proposto por Parasuraman e seus colegas. Neste gráfco identificam-se cinco diferenças (gap) que podem ocorrer na prestação de

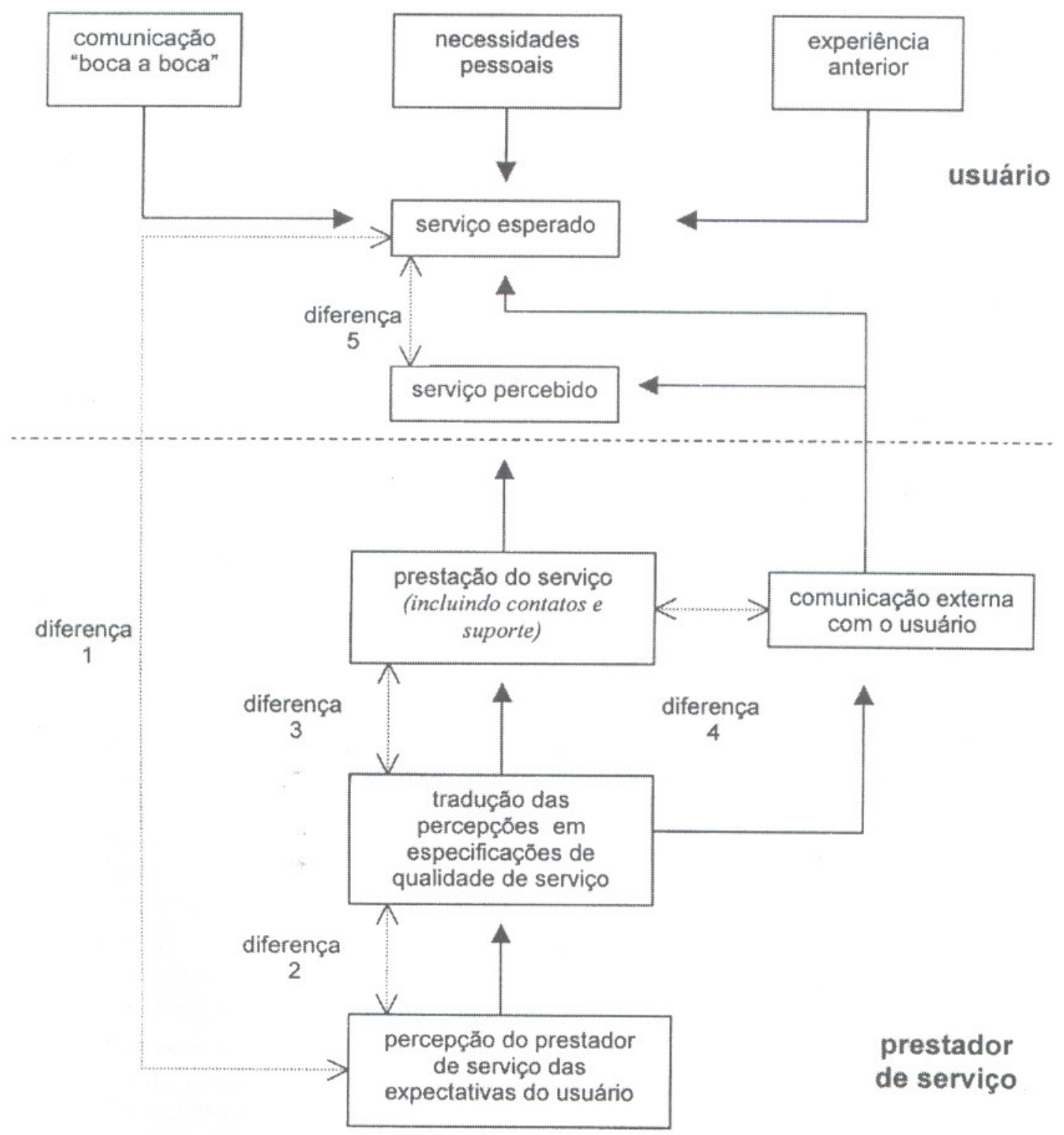

Figura 2. Modelo de qualidade de serviço (gap model). 
serviço. A primeira discrepância ocorre entre o serviço esperado pelo usuário, ou seu ideal de serviço, e a percepção que o prestador do serviço possui da expectativa do usuário. Esta percepção é traduzida em especificações de qualidade (segunda diferença) que gera a prestação de serviço, incluindo contatos prévios e atendimento posterior à prestação de serviço (terceira diferença). Na quarta discrepância temos o efeito da propaganda e comunicação com o usuário em relação ao serviço prestado; trata-se do fenômeno de gerar expectativas infundadas, prometendo o que não é possivel cumprir. Finalmente, tem-se a diferença entre o serviço esperado e o serviço ofertado, gerando a quinta discrepância. Esta diferença entre a expectativa e a percepção é o ponto central da avaliação de Parasuraman, Zeithaml e Berry (1985). A qualidade que um usuário percebe em um serviço está no balanço que este faz entre sua expectativa e o serviço prestado.

\section{DETERMINANTES DA QUALIDADE DE SERVIÇO}

Parasuraman, Zeithaml e Berry (1985) propuseram dez categorias que agrupam os critérios de determinação da qualidade de serviço: confiabilidade, atenção, competência, acessibilidade, cortesia, comunicação, credibilidade, segurança, compreensão, tangibilidade. Em 1988, Parasuraman, Zeithaml e Berry reagruparam estes critérios em cinco dimensões:

I) tangibilidade - facilidades físicas, equipamentos, aparência do pessoal;

II) confiabilidade - capacidade de executar o serviço prometido com confiança e exatidão;

III) atenção - boa vontade em ajudar o usuário e prestar serviço imediato;

IV) segurança - conhecimento e cortesia dos prestadores de serviço e sua capacidade de inspirar confiança;

V) empatia - cuidado, atenção individualizada que a organização presta a seus usuários.

\section{SERVQUAL}

SERVQUAL é um instrumento, proposto por Parasuraman, Zeithaml e Berry (1988; Parasuraman, Berry e Zeithaml,1991b) que mede a expectativa e a percepção de qualidade de serviço em cinco dimensões de atributos. $\mathrm{O}$ instrumento utiliza escala Likert que associa valores numéricos a expressões de apreciação qualitativa do tipo "pouco favorável" a "muito favorável" (Sekaran,
1992, p.170). O SERVQUAL está organizado em três partes: a primeira parte contém 22 questões para medir a expectativa do indivíduo em relação a um dado tipo de serviço; na segunda parte tem-se as mesmas questões, mas direcionadas à avaliação de um determinado serviço prestado; a última parte é uma única pergunta sobre a qualidade do serviço como um todo.

A qualidade de cada dimensão e do serviço como um todo é medida a partir da diferença entre os valores obtidos na primeira parte (expectativa) e a segunda parte (percepção), configurando-se a formulação de qualidade como "Q = P - E", onde "Q" é a qualidade, "E" é a expectativa e "P" é a percepção do serviço. O uso de diferentes escores para depurar um escala de múltiplos itens foi inspirado no trabalho de Ford, Walker e Churchill (1975, citado em Parasuraman, Zeithaml e Berry, 1988).

\section{QUALIDADE DE SERVIÇO EM SISTEMAS DE INFORMAÇÃO}

A avaliação do serviço em sistemas de informação, com abordagem de marketing de serviço, é introduzida por Pitt, Watson e Kavan (1995; cf Pitt, Watson e Kavan,1997; Pitt e Watson, 1994), Ferguson e Zawacki(1993), bem como por Kettinger e Lee (1997,1995, 1994; Kettinger, Lee e Lee, 1995), a partir do trabalho de Parasuraman,Zeithaml e Berry (1985, 1988; Parasuraman, Berry e Zeithaml, 1991b). Myers, Kappelman e Prybutok (1997) acrescentaram ao modelo de DeLone e McLean (1992) este aspecto como uma dimensão, alinhando-o com qualidade da informação.

Outros trabalhos apresentaram o uso do SERVQUAL para avaliação de serviço em bibliotecas e serviços de informação (Rowley, 1996; Edwards e Browne, 1995; Hébert, 1994).

O trabalho de Pitt, Watson e Kavan (1995) foi questionado por Van Dyke, Kappelman e Prybutok (1997) que argumentaram sobre limites da aplicação do instrumento SERVQUAL no contexto de sistemas de informação, iniciando um debate que foi replicado por Pitt, Watson e Kavan (1997) e ponderado por Kettinger e Lee (1997). O problema central nesta questão é o de adaptar o instrumento ao contexto de sistemas de informação, melhorando sua fidedignidade.

Este debate reflete e se baseia em outras discussões realizadas entre o grupo idealizador do instrumento SERVQUAL e outros autores da área de marketing de serviço. Estes debates questionaram a necessidade do instrumento precisar da expectativa do usuário para obter-se a avaliação de qualidade (Babakus 
e Mangold, 1992; Cronin e Taylor, 1992, 1994), a interpretação e operacionalização das expectativas (Teas, 1993, 1994), a confiabilidade e validade da diferença entre expectativa e percepção como formulação de indicador de qualidade (Babakus e Boller, 1992; Brown, Churchill e Peter, 1993) e a dimensionalidade do SERVQUAL (Carman, 1990; Finn e Lamb, 1991). Em resposta a estes questionamentos, os autores apresentaram contra-argumentos, clarificações, explicações e evidências adicionais para reafirmar o valor prático e a confiabilidade do instrumento proposto (Parasuraman, Zeithaml e Berry, 1994b, 1994c; Parasuraman, Berry e Zeithaml, 1993,1991b).

\section{CONCLUSÃO}

Desta revisão é possivel realçar alguns pontos fundamentais: definição das dimensões de um sistema de informação, sistematização das variáveis de qualidade da informação e identificação de uma metodologia para avaliação.

O exame da literatura relacionada a avaliação de sistemas de informação deixa claro que há diferentes maneiras de avaliação e inúmeras variáveis de desempenho. Tendo-se avançado na identificação das dimensões, ainda não se estabeleceu uma sistematização aceitável das variáveis e ainda não se definiu um padrão de avaliação que permita a comparação dos estudos e o estabelecimento de indicadores confiáveis no processo de avaliação de sistemas de informação.

Os autores são unânimes na constatação da dificuldade em se avaliar sistemas de informação, mais ainda as dimensões de qualidade da informação, qualidade de serviço e impacto de sistemas de informação.

O trabalho interdisciplinar se faz necessário para realizar-se aproximações metodológicas que permitam identificar variáveis, modelizar métodos de avaliação, coletar e interpretar dados, bem como analisar e comparar resultados. O campo de pesquisa de avaliação de sistemas de informação é relativamente novo e só recentemente conseguiu-se sistematizar algumas dimensões e busca-se ainda identificar os padrões de mútua influência entre elas.

A contribuição da área de marketing de serviço tem-se mostrado positiva, mas deve ser acompanhada com atenção para que as adaptações e inovações necessárias à área de sistemas de informação sejam realizadas a contento.
O surgimento de novos estudos possibilita a inclusão de novas dimensões que agrupam diferentes fatores que permitem a avaliação de sistemas de informação. Uma nova dimensão no nível de impacto se faz necessária incluir: impacto social, ou impacto socioambiental. Compreendendo-se os sistemas de informação como sistemas abertos, é de prever e desejar que estes desenvolvam interações com o sistema social que os suporta e justifica; isto no entanto é proposta para pesquisas futuras.

\section{REFERÊNCIAS BIBLIOGRÁFICAS}

1. AHIRE, Sanjay L., GOLHAR, Damodar Y., WALlER, Matthew A. Development and validation of TQM implementation constructs. Decision Sciences, v.27, p.23-56. 1996.

2. AHITUV, Niv. A systematic approach toward assessing the value of an information system. MIS Quarterly, v.4, p.61-75.1980.

3. ARORA, Raj, STONER, Charles. The effect of perceived service quality and name familiarity on the service selection decision. The Journal of Services Marketing, v.10, n.1, p.22-34.1996.

4. ASUBONTENG, Patrick, MCCLEARY, Karl J., SWAN, John E. SERVQUAL revisited: a critical review of service quality. The Journal of Services Marketing, v.10, n.6, p.62-81.1996.

5. BABAKUS, Emin, BOLLER, Gregory W. An empirical assessment of SERVQUAL scale. Journal of Business Research, v.24, p.253-268.1992.

6. BABAKUS, Emin, MANGOLD, Glynn W. Adapting the SERVQUAL scale to hospital services: an empirical investigation. Health Services Research, v.26, p.767-786. 1992.

7. BAILEY, James E., PEARSON, Sammy W. Development of a tool for measuring and analyzing computer user satisfaction. Management Science, v.29, p.530-545. 1983.

8. BALL, Leslie, HARRIS, Richard. SMIS members: a membership analysis. MIS Quarterly, v.6, p.19-38.1982.

9. BARKI, Henri, HARTWICK, Jon. Measuring user participation, user involvement, and user attitude. MIS Quarterly, p.59-82.1994.

10. BAROUDI, Jack J., ORLIKOWSKI, Wanda J. A short-form measure of user information satisfaction : psychometric evaluation and notes on use. Journal of Management Information Systems, v.4, p.44-59, Spring. 1988.

11. BERRY, Leonard L., PARASURAMAN, A. Building a new academic field: the case of services marketing. Journal of Retailing, v.69, p.13-60.1993. 
12. BERRY, Leonard L., PARASURAMAN, A. Prescriptions for a service quality revolution in America. Organizational Dynamics, v.20, p.5-15.1992.

13. BERRY, Leonard L., PARASURAMAN, A. Marketing services: competing through quality. New York: Free Press, 1991.

14. BERRY, Leonard L., PARASURAMAN, A., ZEITHAML, Valarie A. Improving service quality in America: lessons learned, executive commentary. Academy of Management Executive, v.8, p.32-52.1994.

15. BERRY, Leonard L., PARASURAMAN, A., ZEITHAML, Valarie A. Ten lessons for improving service quality. Cambridge, Massachusetts: Marketing Science Institute, 1993. 25p. (MSI Report, 93-104).

16. BERRY, Leonard L., ZEITHAML, Valarie A., PARASURAMAN, A. Five imperatives for improving service quality. Sloan Management Review, p29-38. 1990.

17. BERRY, Leonard L., PARASURAMAN, A., ZEITHAML, Valarie A. The service-quality puzzle. Business Horizons, v.31, p.35-43.1988

18. BERRY, Leonard L., PARASURAMAN, A., ZEITHAML, Valarie A. Synchronizing demand and supply in service businesses. Business, v.34, p.35-37.1984.

19. BLAYLOCK, Bruce K., REES, Loren P. Cognitive style and the usefulness of information. Decision Sciences, v.15, p. 74-91.1984.

20. BOUGHTON, Paul D., NOWAK, Linda, WASHBURN, Judith. A decision model for marketing research relationship choices. The Journal of Services Marketing, v.10, n.1, p. 56-69.1996.

21. BRANCHEAU, James C., WETHERBE, James C. Key issues in information systems management. MIS Quarterly, v.11, p.23-45.1987.

22. BRANCHEAU, James C., JANZ, Brian D., WETHERBE, James C. Key issues in information systems management:1994-1995 SIM Delphi results. MIS Quarterly, v.20, p.225-242.1996.

23. BROWN, Tom J., CHURCHILL JR., Gilbert A., PETER, J. Paul. Improving the measurement of service quality. Journal of Retailing, v.69, p.127-139.1993.

24. CARMAN, James M. Consumer perceptions of service quality: an assessment of the SERVQUAL dimensions. Journal of Retailing, v.66, p.33-55.1990.

25. COE, Larry R. Five small secrets to systems success. Information Resources Management Joumal, v.9, p. 29-38.1996.

26. CONRATH, David W., MIGNEN, Olivier P. What is being done to measure user satisfaction with EDP/MIS. Information \& Management, v.19, p.7-19.1990.

27. CRONIN JR., J. Joseph, TAYLOR, Steven A. SERVPEF versus SERQUAL: reconciling performance-based and perceptions-minus-expectations measurement of service quality. Journal of Marketing, v.58, p.125-131.1994.
28. CRONIN JR., J. Joseph, TAYLOR, Steven A. Measuring service quality: a reexamination and extension. Journal of Marketing, v.56, p.55-68.1992.

29. DAVENPORT, Thomas H., BEERS, Michael C. Managing information about processes. Journal of Management Information Systems, v.12, p.57-80, Summer. 1995.

30. DELONE, William H. Determinants of success for computer usage in small business. MIS Quarterly, p. $51-61.1988$.

31. DELONE, William H., MCLEAN, Ephraim R. Information system success: the quest for dependent variable. Information Systems Research, v.3, p. 60-95.1992.

32. DICKSON, Gary W., WETHERBE, James C. Management assessment and evaluation of MIS. In: DICKSON, Gary W., WETHERBE, James C. The management of information systems. New York: McGraw-Hill,1983. p.162-186.

33. DICKSON, Gary W., LEITHEISER, Robert L., WETHERBE, James C., et al. Key information systems issues for the 1980's. MIS Quarterly, v.8, p.135-159.1984.

34. EDBERG, Dana T., BOWMAN, Brent J. User-developed applications: an empirical study of application quality and developer productivity. Journal of Management Information Systems, v.13, p.167-185, Summer.1996.

35. EDWARDS, Susan, BROWNE, Mairéad. Quality in information services: do users and librarians differ in their expectations? Library \& Information Science. Research, v.17, p.163-183.1995.

36. ETEZADI-AMOLI, Jamshid, FARHOOMAND, Ali F. A structural model of end user computing satsfaction and user performance. Information \& Management, v.30, p.65- 73.1996 .

37. FERGUSON, J. M., ZAWACKI, R. A. Service quality : a critical success factor for IS organizations. Information Strategy : the executive's journal, v.9, p.24-30.1993.

38. FIEDLER, Kirk Dean, GROVER, Varun, TENG, James T. C. An empirical derived taxonomy of information technology structure and its relationship to organizational structure. Journal of Management Information Systems, v.13, p.9-34, Summer.1996.

39. FINN, David W., LAMB JR., Charles W. An evaluation of the SERVQUAL scales in a retail setting. In: HOLMAN, Rebecca H., SOLOMON, Michael R. Advance in consumer research. Provo: Association for Consumer Research,1991.p.

40. FISK, Raymond P., BROWN, Stephen W., BITNER, Mary Jo. Tracking the evolution of the services marketing literature. Journal of Retailing, v.69, p.61-103.1993.

41. FORD, Neil M., WALKER JR., Orville C., CHURCHILL JR., Gilbert A. Expectation-specifc measures of the intersender conflict and role ambiguity experienced by industrial salesmen. Journal of Business Research v.3, p.95-112.1975.

42. FREITAS, Henrique M. R. de, BALLAZ, Bernard, MOSCAROLA, Jean. Avaliação de sistemas de informações. 
Revista de Administração, São Paulo, v. 29, n. 4, p. 36-55. 1994.

43. GALLAGHER, Charles A. Perceptions of the value of a management information system. Academy of Management Journal, v.17, p.46-55.1974.

44. GAllettA, Denis F., LEDERER, Albert L. Some cautions on the measurement of user information satisfaction. Decision Sciences, v.20, p.419-438.1989.

45. GROVER, Varun, JEONG, Seung Ryul, SEGARS, Albert $H$. Information systems effectiveness: the construct space and patterns of application. Information \& Management, v.31, p.177-191.1996.

46. GUYNES, Carl Stephen, VANECEK, Michael T. Critical success factors in data management. Information \& Management, v.30, p.201-209.1996.

47. HAMILTON, Scott, CHERVANY, Norman L. Evaluating information system effectiveness - part I: comparing evaluation approaches. MIS Quarterly, v.5, p.55-69. 1981.

48. HÉBERT, Françoise. Service quality: an unobtrusive investigation of interlibrary loan in large public libraries in Canada. Library \& Information Science Research v.16, p.3-21.1994.

49. HUI, Michael K., TSE, David K. What to tell consumers in waits of different lengths: an integrative model of service evaluation. Journal of Marketing, v.60, p.81-90.1996.

50. IIVARI, Juhani, KOSKELA, Erkki. The PIOCO model for information systems design. MIS Quarterly, v.11, p.401-419.1987.

51. ISHMAN, Michael D. Measuring information success at the individual level in cross-cultural environments. Information Resources Management Journal, v.9, p. 16-28. 1996.

52. IVES, Blake, OLSON, Margrethe. User involvement and and MIS success: a review of research. Management Science, v.30, p.586-603. 1984

53. IVES, B., OLSON, M., BAROUDI, Jack J. The measurement of user information satisfaction. Communications of the ACM, v.26, p.785-793.1983.

54. JARVENPAA, Sirkka L., DICKSON, Gary W., DESANCTIS, Gerardine. Methodological issues in experimental IS research: experiences and recommendations. MIS Quarterly, v.9, p.141-156.1985.

55. JENSEN, John B., MARKLAND, Robert E. Improving the application of quality conformances tools in service firms. The Journal of Services Marketing, v.10, n.1, p.35-55. 1996.

56. JONES, Jack William, MCLEOD JR., Raymond. The structure of executive information systems: an exploratory analysis. Decision Sciences, v.17, p.220-249.1986.

57. JOSHI, Kailash. A model of users' perspective on change: the case of information systems technology implementation. MIS Quarterly, v.15, p.229-242.1991.
58. JOSHI, Kailash. An investigation of equity as a determinant of user information satisfaction. Decision Sciences, v.21, p.786-807.1990.

59. KEEN, Peter G. W. MIS research: reference disciplines and a cumulative tradition. In: ANON. Proceedings of the first International Conference on Information Systems (ICIS), 1980. p.220-232.

60. KETTINGER, William J., LEE, Choong C. Pragmatic perspectives on the measurement of information systems service quality. MIS Quarterly, v.21, p.223-240.1997.

61. KETTINGER, William J., LEE, Choong C. Exploring a "gap" model of information services quality. Information Resources Management Journal, v.8, p.5-16.1995.

62. KETTINGER, William J., LEE, Choong C. Perceived service quality and user satisfaction with the information services function. Decision Sciences, v.25, p.737-766. 1994.

63. KETtinger, William J., LEE, Choong C., LEE, Sunro. Global measures of information service quality: a cross-national study. Decision Sciences, v.26, p.569-588. 1995.

64. KING, William R., EPSTEIN, Barry J. Assessing information system value. Decision Sciences, v.14, p.34-45. 1983.

65. KLEIN, Barbara D., GOODHUE, Dale L., DAVIS, Gordon B. Can humans detect errors in data? impact of base rates, incentives, and goals. MIS Quarterly, v.21, p.169-194. 1997.

66. KRAEMER, Kenneth L., DANZIGER, James N., DUNKLE, Debora E., et al. The usefulness of computerbased information to public managers. MIS Quarterly, v.17, p.129-147.1993.

67. LARCKER, David F., LESSIG, V. Parker. Perceived usefulness of information: a psychometric examination. Decision Sciences, v.11, p.121-134.1980.

68. LEONARD, Myron. Marketing literature review. Journal of Marketing, v.60, p.121-133. 1996.

69. MAGAL, Simha R., CARR, Houston H., WATSON, Hugh J. Critical success factors for information center managers. MIS Quarterly, v.12, p.413-425. 1988.

70. MAHMOOD, Mo A. systems development methods: a comparative investigation. MIS Quarterly, v.11, p.293-311. 1987.

71. MAHMOOD, Mo A., MEDEWITZ, Jeanette N. Impact of design methods on decision support systems success: an empirical assessement. Information \& Management, v.9, p.137-151.1985.

72. MARKUS, Lynne, ROBEY, Daniel. Information technology and organizational change: conceptions of causality in theory and research. Management Science, v.34, p.583-598.1988.

73. MASON, Richard O. Measuring information output: a communication systems approach. Information \& Management, v.1, p.219-234.1978. 
74. MELONE, Nancy Paule. A theoretical assessment of the user-satisfaction construct in information systems research. Management Science, v.36, p.76-91.1990.

75. MEYER, Marc H., ZACK, Michael H. The design and development of information products. Sloan Management Review, v.37, p.43-59.1996.

76. MILLER, J., DOYLE, B. A. Measuring effectiveness of computer based information systems in the financial services sector. MIS Quarterly, v.11, p.107-124.1987.

77. MONTAZEMI, Ali R., CAMERON, David A., GUPTA, Kalyan Moy. An empirical study of factors affecting software package selection. Journal of Management Information Systems, v.13, p.89-105, Summer.1996.

78. MORGADO, Eduardo Martins, WATSON, Richard T. Qualidade dos serviços na área de informática: um modelo para avaliação. cadernos de Gestão dos Sistemas e Tecnologias da Informação "Henrique Marcelino", n. 6,1998.

79. MORGAN, Robert M., HUNT, Shelby D. The commitment-trust theory of relationship marketing. Journal of Marketing, v.58, p.20-38.1994.

80. MYERS, Barry L., KAPPELMAN, Leon A., PRYBUTOK, Victor R. A comprehensive model for assessing the quality and productivity of the information systems function: toward a theory for information systems assessment. Information Resources Management Journal, v.10, p.6-25.1997.

81. NIEDERMAN, Fred, BRANCHEAU, James C., WETHERBE, James C. Information systems management issues for the 1990's. MIS Quarterly, v.15, p.475-500.1991.

82. OLSON, Margrethe H., LUCAS, Henry C. The impact of office automation on the organization: some implications for research and practice. Communications of the ACM, v.25, p.838-847.1982.

83. PALVIA, Prashant C. A model and instrument for measuring small business user satisfaction with information technology. Information \& Management, v.31, p. 151-163. 1996.

84. PARASURAMAN, A., BERRY, Leonard L. Marketing services: competing through quality. New York / Toronto: Free Press / Maxwell Macmillan Canada,1991. 212p.

85. PARASURAMAN, A., ZEITHAML, Valarie A., BERRY, Leonard L. Alternative scales for measuring service quality: a comparative assessment based on psychometric and diagnostic criteria. Journal of Retailing, v.70, p.201$230.1994 \mathrm{a}$

86. PARASURAMAN, A., ZEITHAML, Valarie A., BERRY, Leonard L. Moving foward in service quality research: measuring different customer-expectation levels, comparing alternative scales, and examining the performance-behavioral intentions link. Cambridge, Massachusetts: Marketing Science Institute,1994b. 54p. (MSI Report, 94-114).

87. PARASURAMAN, A., ZEITHAML, Valarie A., BERRY, Leonard L. Reassessment of expectations as a comparison standard in measuring service quality: implications for futher research. Journal of Marketing, v. 58 , p.111-124.1994c.

88. PARASURAMAN, A., BERRY, Leonard L., ZEITHAML, Valarie A. More on improving the measurement of service quality. Journal of Retailing, v.69, p.140-147. 1993.

89. PARASURAMAN, A., BERRY, Leonard L., ZEITHAML, Valarie A. Perceived service quality as a customer-based performance measure: an empirical examination of organizational barries using an extended service quality model. Human Resource Management, v.30, p.335364.1991a.

90. PARASURAMAN, A., BERRY, Leonard L., ZEITHAML, Valarie A. Refrinement and reassessment of the SERVQUAL scale. Journal of Retailing, v.67, p.420$450.1991 b$.

91. PARASURAMAN, A., BERRY, Leonard L., ZEITHAML, Valarie A. Understanding customer expectations of service. Sloan Management Review, v.32, p.39-48. $1991 \mathrm{c}$

92. PARASURAMAN, A., BERRY, Leonard L., ZEITHAML, Valarie A. An empirical examination of relationships in an extended service quality model. Cambridge, Massachusétts: Marketing Science Institute, 1990. 56p. (MSI Report, 90-122).

93. PARASLTRAMAN, A., ZEITHAML, Valarie A., BERRY, Leonard L. SERVQUAL: a multiple-item scale for measuring costumer perceptions of service quality. Journal of Retailing, v.64, p.12-40.1988.

94. PARAUTRAMAN, A., ZEITHAML, Valarie A., BERRY, Leonard L. A conceptual model of service quality and its implications for future research. Journal of Marketing, v.49, p.41-50.1985.

95. PARSONS, Jeffrey. An information model based on classification theory. Management Science, v.42, p.1437-1453. 1996.

96. PITT, Leyland F., WATSON, Richard T. Longitudinal measurement of service quality in information systems: a case study. In: Proceedings of the fifteenth International Conference on Information Systems (ICIS). Vancouver, 1994, p.419-428.

97. PITT, Leyland F., WATSON, Richard T., KAVAN, C. Bruce. Measuring information systems service quality: concerns for a complete canvas. MIS Quarterly, v.21, p.209-221.1997

98. PITT, Leyland F., WATSON, Richard T., KAVAN, C. Bruce. Service quality: a measure of information systems effectiveness. MIS Quarterly, p.173-185.1995.

99. POWPAKA, Samart. The role of outcome quality as determinant of overall service quality in different categories of services industries: an empirical investigation. The Journal of Services Marketing, v.10, n.2, p.5-25.1996. 
100. RAINER JR., R. K., WATSON, H. J. The keys to executive information system success. Journal of Management Information Sstems, v.12, p.83-98.1995.

101. RIVARD, Suzanne, HLTFF, Sid L. An empirical study of users as application developers. Information \& Management, v.8, p.89-102.1985.

102. RIVARD, Suzanne, HUFF, Sid L. User development applications: evaluation of success from the DP departament perspective. MIS Quarterly, v.8, p.39-50.1984.

103. ROWLEY, Jennifer. Managing quality in information services. Information Services \& Use, v.16, p.51-61.1996.

104. RUSHINEK, Avi, RUSHINEK, Sara F. What makes users happy? Communications of the ACM, v.29, p.594-598. 1986.

105. SAARINEN, Timo. An expanded instrument for evaluating information system success. Information \& Management, v.31, p.103-118.1996.

106. SALEEM, Naveed. An empirical test of the contingency approach to user participation in information systems development. Journal of Management Information Systems, v.13, p.145-166, Summer.1996.

107. SAUNDERS, Carol Stoak, JONES, Jack William. Measuring performance of the information systems function. Journal of Management Information Systems, v.8, p.63-82, Spring. 1992.

108. SEDDON, Peter, KIEW, Min-Yen. A partial test and development of the DeLone and McLean model of IS success. In:. Fifteenth Annual Conference on Information Systems (ICIS), 1994. p.99-110.

109. SEKARAN, Uma. Research methods for business: a skill-building approach. 2.ed. New York: John Wiley and sons, 1992. 428p.

110. SENGE, Peter M. The leader's new work: building learning organisations. Sloan Management Review, p. 7-23.1990

111. SHANnON, Claude Elwood, WEAVER, Warren. The mathematical theory of communication. Urbana: University of Illinois Press, 1949.

112. SPRAGUE JR., Ralph H. Electronic document management: challenges and opportunities for information systems managers. MIS Quarterly, p.29-49.1995.

113. SPRENG, Richard A., MACKENZIE, Scott B., OLSHAVSKY, Richard W. A reexamination of the determinants of consumer satisfaction. Journal of Marketing, v.60, p.15-32.1996.

114. SRINIVASAN, Ananth. Alternative measures of system effectiveness: assossiacions and implications. MIS Quarterly, v.9, p.243-253.1985.

115. SWANSON, E. Burton. Information channel disposition and use. Decision Sciences, v.18, p.131-145.1987.
116. SWANSON, E. Burton. Management information systems: appreciation and involvement. Management Science, v.21, p.178-188.1974.

117. TEAS, R. Kenneth. Expectations as a comparison standard in measuring service quality: an assessment of a reassessment. Journal of Marketing, p.132-139.1994.

118. TEAS, R. Kenneth. Expectations, performance evaluation, and customers' perceptions of quality. Journal of Marketing, v.57, p.18-34.1993.

119. VAN DYKE, Thomas P., KAPPELMAN, Leon A., PRYBUTOK, Victor R. Measuring information systems service quality: concerns on the use of the SERVQUAL questionnaire. MIS Quarterly, v.21, p.195-208.1997.

120. WEBSTER, C. Can customers be segmented on the basis of their service quality expectations? The Journal of Services Marketing, v.3, n.2, p.35-53.1989.

121. WEITZEL, William, SCHWARZKOPF, Albert B., PEACH, E. Brian. The influence of customer service on retail stores. Journal of Retailing, v.65, p27-39.1989.

122. YVES, Blake, HAMILTON, Scott, DAVIS, Gordon B. A framework for research in computer-based management information systems. Management Science, v.26, p.910-934. 1980.

123. ZEITHAML, Valarie A., BERRY, Leonard L., PARASURAMAN, A. The behavioral consequences of service quality. Journal of Marketing, v.60, p.31-46.1996.

124. ZEITHAML, Valarie A., BERRY, Leonard L., PARASURAMAN, A. The nature and determinants of customer expectations of service. Journal of the Academy of Marketing Science, v.21, n.1, p.1-12.1993.

125. ZEITHAML, Valarie A., BERRY, Leonard L., PARASUTRAMAN, A. The nature and determinants of costumer expectations of service. Cambridge, Massachusetts: Marketing Science Institute, 1991.27p. (MSI Report, 91-113).

126. ZEITHAML, Valarie A., PARASURAMAN, A., BERRY, Leonard L. Delivering quality service: balancing customer perceptions and expectations. New York: Free Press, 1990.

127. ZEITHAML, Valarie A., BERRY, Leonard L., PARASURAMAN, A. Communication and control processes in the delivery of service quality. Journal of Marketing, v.52, p.35-48.1988.

128. ZEITHAML, Valarie A., PARASURAMAN, A., BERRY, Leonard L. Problems and strategies in services marketing. Journal of Marketing, v.49, p.33-46.1985.

129. ZMUD, Robert W. Individual differences and MIS success: a review of the empirical literature. Management Science, v.25, p.966-979.1979.

130. ZMUD, Robert W. An empirical investigation of the dimensionality of the concept of information. Decision Sciences, v.9, p.187-195.1978. 